\title{
Sulfate enhances drought tolerance in foxtail millet seedlings by promoting ABA biosynthesis and inducing stomatal closure
}

\section{Yansha Han}

Shanxi University

Dianqing Gong

Taiyuan University of Technology

Huilan Yi ( $\nabla$ yihl@sxu.edu.cn )

Shanxi University https://orcid.org/0000-0002-9298-8313

\section{Research Article}

Keywords: Foxtail millet, Sulfate, Drought tolerance, ABA, Stomatal closure

Posted Date: August 6th, 2021

DOI: https://doi.org/10.21203/rs.3.rs-779538/v1

License: (c) This work is licensed under a Creative Commons Attribution 4.0 International License.

Read Full License 


\section{Abstract \\ Background and aims}

Sulfate, the main source of sulfur in natural soil, is critical for plant growth and development, as well as plant responses to environmental stress, including drought. However, our understanding of the detailed mechanisms of sulfate-modulated drought tolerance in crop plants is far from complete. In the present study, by using foxtail millet (Setaria italica L.), an emerging model crop, we investigated the possible mechanisms involved in sulfate-induced crop tolerance to drought stress.

\section{Methods}

A combination of biochemical and molecular approaches, as well as stomatal apertures analyses were applied to determine the effect of sulfate application on sulfur assimilation, ABA biosynthesis, and stomatal movement.

\section{Results}

Upon drought exposure, sulfate $(4 \mathrm{mM})$ pretreatment significantly alleviated the decrease in relative water content in foxtail millet leaves. Exogenous sulfate increased endogenous sulfate content and markedly enhanced the enzyme activity of sulfite reductase (SiR) and $O$-acetylserine(thiol)lyase (OASTL), as well as levels of their transcripts, leading to an increase in cysteine (Cys) production in drought-stressed leaves. Furthermore, in comparison with drought stress alone, sulfate application significantly upregulated the transcriptional expression of SIABA3 and SIAAO3, which contributed to the increased ABA levels in the leaves of drought-stressed foxtail millet seedlings. Moreover, the addition of sulfate decreased stomatal aperture, thus resulting in reduced leaf water loss in foxtail millet exposed to drought.

\section{Conclusion}

Our data suggest that sulfate application was able to promote drought tolerance of foxtail millet plants, at least partially by increasing ABA biosynthesis and triggering stomatal closure.

\section{Introduction}

Drought is one of the major environmental stresses severely limiting agricultural productivity worldwide. Drought stress results in diverse physiological and biochemical changes, such as dramatic water loss, reduced photosynthetic efficiency, and impaired nutrient metabolism, ultimately inhibiting plant growth and development and even leading to premature plant death (Xu et al. 2010; Basu et al. 2016). Plants have evolved multiple strategies to minimize the adverse impacts of drought stress, including structural 
alteration, osmotic adjustment, and activation of antioxidant defences (Xu et al. 2010; Qi et al. 2018; Du et al. 2019).

Stomata on leaf surfaces control gas exchange and water transpiration in plants (Qi et al. 2018). Reducing transpirational water loss through stomata is one of the main regulatory mechanisms in plant adaptation to drought stress (Gupta et al. 2020). It is generally accepted that under drought conditions, accumulation of the hormone abscisic acid (ABA) in guard cells induces stomatal closure to limit water loss via transpiration (Lim et al. 2015). ABA is synthesized from C40-carotenoid precursors in plastids, which are oxidatively cleaved by 9-cis-epoxycarotenoid dioxygenase (NCED) to produce the C15 compound xanthoxin (Milborrow 2001). Xanthoxin is further transformed into abscisic aldehyde, which is oxidized by abscisic aldehyde oxidase (AAO3) to form ABA in the cytoplasm (Milborrow 2001).

Sulfur (S) is an essential element for plants, as it modulates a wide variety of biological processes, including plant growth, development, and stress responses (Kopriva et al. 2019). Sulfate taken up from soil by roots is the main source of sulfur for plants and must be reduced to cysteine (Cys) before entering other metabolic pathways (Koralewska et al. 2009; Takahashi 2019). Within the plastids of plant cells, sulfate is activated by ATP sulfurylase (ATPS) to produce adenosine 5 '-phosphosulfate (APS), followed by reduction to sulfite. Sulfite is then reduced by sulfite reductase $(\mathrm{SiR})$ to form sulfide, which is further incorporated into Cys by the action of $O$-acetylserine(thiol)lyase (OASTL; Kopriva et al. 2019). Previous studies have shown that exogenously applied sulfate promotes sulfur assimilation and stimulates the biosynthesis of sulfur-containing compounds, including glutathione and phytochelatins, thereby enhancing heavy metal detoxification in both woody and herbaceous plants (Liang et al. 2016; Ma et al. 2018). Under water deficit conditions, sulfate application can improve drought tolerance in maize by promoting antioxidant defence and increasing photosynthetic activity (Usmani et al. 2020). ABA has traditionally been considered a primary root-to-shoot signal of water stress (Schachtman and Goodger 2008). Interestingly, however, Ernst et al. (2010) reported that sulfate served as an early xylem-delivered chemical signal during soil drying preceding the biosynthesis of root-sourced ABA. In poplar plants, drought stress has been shown to enhance xylem sap sulfate levels by decreasing sulfate xylem uploading and increasing sulfate efflux from xylem parenchyma cells (Malcheska et al. 2017). Furthermore, supplying sulfate via the petioles decreased stomatal conductance in detached poplar leaves (Malcheska et al. 2017). Remarkably, recent studies with Arabidopsis have demonstrated that sulfate is incorporated into Cys to trigger ABA synthesis and promote stomatal closure in leaves (Batool et al. 2018; Rajab et al. 2019). However, for cereal crops, the interaction between sulfate and ABA during the drought response has not been fully elucidated.

Foxtail millet (Setaria italica L.) is a $\mathrm{C}_{4}$ cereal crop widely grown in the arid and semiarid regions of northern China (Pant et al. 2016; Hao et al. 2020). In recent years, with the completion of whole-genome sequencing, foxtail millet has emerged as a model system for studying $\mathrm{C}_{4}$ plant biology, including agronomic traits and stress responses (Zhang et al. 2012a; Tang et al. 2017; Yang et al. 2020). This study aims to determine the potential effect of sulfate supply on drought stress responses in foxtail millet seedlings. We hypothesized that exogenously applied sulfate may increase ABA levels and thus trigger 
stomatal closure in the leaves under drought conditions. To test this hypothesis, we examined sulfur assimilation, ABA biosynthesis, and stomatal movement in drought-stressed foxtail millet plants. The results obtained are important for further elucidation of the role of sulfate in regulating plant responses to drought and for exploring effective strategies to enhance the drought tolerance of cereal crops.

\section{Materials And Methods}

\section{Plant cultivation}

Seeds of foxtail millet (Setaria italica, ecotype Changnong44) were soaked in distilled water for $12 \mathrm{~h}$ at 25 ${ }^{\circ} \mathrm{C}$ and then sown in plastic pots $(10 \times 10 \times 8 \mathrm{~cm})$ filled with sterilized nutrient soil. The pots were maintained in a culture room with a 14-h light/10-h dark photoperiod, a day/night temperature of 25 ${ }^{\circ} \mathrm{C} / 18{ }^{\circ} \mathrm{C}, 180 \mu \mathrm{mol} \mathrm{m} \mathrm{m}^{-2} \mathrm{~s}^{-1}$ of photosynthetically active radiation and a relative humidity of $50-60 \%$. After germination, the seedlings were watered every three days.

\section{Experimental treatments}

To examine the effects of sulfate application on the drought tolerance of foxtail millet plants, three-weekold seedlings of uniform size were subjected to various treatments: (1) Seedlings were irrigated with water containing the indicated concentrations of $\mathrm{MgSO}_{4}(50 \mathrm{~mL}$ water per pot). Three days later, the seedlings were subjected to drought stress by withholding watering. After $0,3,5$, and 7 days of water deprivation, the seedlings were photographed, and leaf relative water content (RWC) was assayed. The leaf RWC was determined according to the method of Han et al. (2019). $0 \mathrm{~d}$ was defined as the initiation of drought treatment. (2) Seedlings were irrigated with water or $4 \mathrm{mM} \mathrm{MgSO}_{4}$ solution and then either regularly watered or exposed to drought. The leaf samples were harvested after 0 and 5 days of drought stress for physiological, biochemical and molecular analyses. (3) For the stomatal aperture assay, seedlings were divided into four groups: a) control; b) sulfate; c) ABA; and d) sulfate + ABA. For the control and sulfate treatments, the seedlings were each irrigated with water or $4 \mathrm{mM} \mathrm{MgSO}_{4}$ solution. For the sulfate $+A B A$ and $A B A$ treatments, the seedlings were foliar sprayed with $5 \mu \mathrm{M} A B A$ with or without 4 $\mathrm{mM} \mathrm{MgSO}_{4}$ irrigation. Then, in each group, half of the seedlings were regularly watered, while the other half were subjected to drought stress by withholding watering for 5 days.

\section{Sulfate and Cys content assays}

Sulfate content was assayed by the method described by Chen et al. (2019) with some modifications. In brief, the leaf samples were homogenized in $10 \mathrm{mM} \mathrm{HCl}$ and then centrifuged at $12,000 \mathrm{~g}$ for $15 \mathrm{~min}$. Two millilitres of each supernatant was mixed with $0.35 \mathrm{~mL}$ of $0.5 \mathrm{mM} \mathrm{HCl}$ and $0.15 \mathrm{~mL}$ of $0.1 \mathrm{M} \mathrm{BaCl}_{2}$. After incubation for $5 \mathrm{~min}$ at $25^{\circ} \mathrm{C}$, the absorbance at $410 \mathrm{~nm}$ was determined using $\mathrm{K}_{2} \mathrm{SO}_{4}$ as a standard.

Cys content was quantified using the acid ninhydrin reagent according to the method of Gaitonde (1967). Measurement of ATPS, SiR, and OASTL activity 
ATPS activity was assayed by measuring molybdate-dependent pyrophosphate (PPi) formation as reported by Liang et al. (2016). Leaf samples were homogenized with $20 \mathrm{mM}$ Tris-HCl buffer (pH 8.0) containing 1\% (w/v) PVP, 2 mM DTT, and $10 \mathrm{mM}$ EDTA. After centrifugation at $16,000 \mathrm{~g}$ and $4{ }^{\circ} \mathrm{C}$ for 10 min, each supernatant was used as an enzyme extract for ATPS activity assays.

For the measurement of SiR activity, leaf samples were homogenized with $50 \mathrm{mM}$ potassium phosphate buffer ( $\mathrm{pH}$ 7.8) and then centrifuged at $12,000 \mathrm{~g}$ and $4{ }^{\circ} \mathrm{C}$ for $20 \mathrm{~min}$. Then, $100 \mu \mathrm{L}$ of each supernatant was added to a separate reaction mixture containing $0.2 \mathrm{mM} \mathrm{NADPH}, 0.5 \mathrm{mM} \mathrm{Na}_{2} \mathrm{SO}_{3}, 0.1 \mathrm{mM} \mathrm{Na}_{2} \mathrm{EDTA}_{\text {, }}$ and $50 \mathrm{mM}$ potassium phosphate buffer ( $\mathrm{pH} 7.8)$. The decrease in absorbance was recorded at $340 \mathrm{~nm}$. One unit $(U)$ of SiR activity was defined as the amount of enzyme that catalysed the oxidation of $1 \mu \mathrm{mol}$ NADPH per minute.

OASTL activity was determined by monitoring the formation of Cys at $560 \mathrm{~nm}$ as described by Liang et al. (2016). Protein concentrations of the enzyme extracts were estimated by the Bradford method using BSA as a standard (Bradford 1976).

\section{Determination of ABA content}

ABA content was measured as previously described (Fang et al. 2017), with some minor modifications. Briefly, fresh leaf samples $(0.1 \mathrm{~g})$ were homogenized with precooled extraction solution ( $80 \%$ methanol, $\mathrm{v} / \mathrm{v}$ ). After incubation at $4{ }^{\circ} \mathrm{C}$ for $2 \mathrm{~h}$, each mixture was centrifuged at $10,000 \mathrm{~g}$ for $20 \mathrm{~min}$ at the same temperature. Each supernatant was passed through a CNWBOND LC-C18 cartridge (CNW Technologies $\mathrm{GmbH}$, Germany). The elution products were dried by rotary evaporation and then dissolved separately in $20 \mathrm{~mL}$ cold solution from an ABA enzyme-linked immunosorbent assay (ELISA) kit (MLBIO, Shanghai, China). The ABA contents were determined using an ELISA kit according to the manufacturer's instructions.

\section{Stomatal aperture bioassay}

The epidermal strips of the third leaf from the tip of the stem of each sampled plant were used for stomatal aperture assays. The abaxial epidermis was peeled off the leaves and immediately mounted on glass slides. Stomata were randomly imaged with an optical microscope. Over 100 stomata were examined from epidermal strips of six individual plants. The widths and lengths of the stomata were quantified using ImageJ software. The stomatal aperture is expressed as the ratio of the width to the length of the stoma.

\section{Water loss rate determination}

The rate of water loss was measured as described by Zhang et al. (2012b). In brief, immediately after the third leaf from the tip was excised from each seedling, the initial fresh weight was measured $\left(\mathrm{FW}_{0}\right)$. The detached leaves were placed on a bench under a light intensity of $150-180 \mu \mathrm{mol} \mathrm{m}{ }^{-2} \mathrm{~s}^{-1}$ at $25^{\circ} \mathrm{C}$ and weighed at different time intervals (FW) over a period of $120 \mathrm{~min}$. The water loss rate of detached leaves was calculated as follows: Water loss rate $(\%)=\left\{\left(\mathrm{FW}_{0}-\mathrm{FW}\right) / \mathrm{FW}_{0}\right\} \times 100$. 


\section{Gene expression analysis}

Total RNA was extracted from leaf samples using TRIzol reagent (TaKaRa, Tokyo, Japan). Transcript expression levels were assayed by quantitative real-time PCR using SYBR Premix Ex Taq II (TaKaRa, Tokyo, Japan) in the Bio-Rad CFX96TM Real-Time System (Bio-Rad, CA, USA). Actin was used as the internal control. Relative expression levels of the target genes were determined using the $2^{-\triangle \triangle C T}$ method (Livak and Schmittgen 2001). The primers are listed in supplementary Table S1.

\section{Statistical analysis}

Data are expressed as the mean values \pm standard errors (SE) of at least three independent replicates. One-way analysis of variance (ANOVA) with Duncan's multiple range test was performed to determine the significant differences among groups using SPSS version 17.0 software $(P<0.05)$.

\section{Results}

\section{Sulfate application enhanced the drought tolerance of foxtail millet seedlings}

Three-week-old foxtail millet seedlings were pretreated with different concentrations of sulfate $(1,4$, and 8 $\mathrm{mM}$ ) and then subjected to drought stress. As shown in Fig. 1A, drought reduced relative water content (RWC) in the leaves of foxtail millet seedlings in a time-dependent manner. At the three time points $(3,5$, and $7 \mathrm{~d}$ ) of drought stress, pretreatment with $4 \mathrm{mM}$ sulfate markedly increased leaf RWC compared with the control, while $1 \mathrm{mM}$ and $8 \mathrm{mM}$ sulfate showed no significant effect (Fig. 1A). Continuous drought treatment caused foliar injury in the growing seedlings. After 5 days of drought stress, the leaves of seedlings started to wilt (Fig. 1B). Stunted growth and even leaf necrosis were clearly observed after 7 days of drought treatment (Fig. 1B). Sulfate application (4 mM) significantly lessened injury symptoms in the drought-stressed seedlings (Fig. 1B). Taken together, these results suggest that $4 \mathrm{mM}$ sulfate was able to enhance drought tolerance in foxtail millet seedlings.

\section{Exogenous sulfate application enhanced sulfur assimilation and increased Cys accumulation in drought-stressed foxtail millet leaves}

After 5 days of drought stress, sulfate content in the leaves of foxtail millet seedlings markedly decreased compared with that of the control (Fig. 2). Exogenous sulfate (4 mM) application led to a significant increase in sulfate content in drought-stressed leaves; i.e., a $66.7 \%$ increase in the sulfate + drought group compared to the drought group after 5 days of withholding water (Fig. 2).

ATPS, SiR, and OASTL are important enzymes that catalyse the conversion of inorganic sulfate into Cys via the sulfur assimilation pathway. Five days of drought stress caused a significant increase in the 
activity of ATPS, SiR, and OASTL relative to the control (Fig. 3A-C). Sulfate pretreatment further enhanced SiR and OASTL enzyme activity in drought-stressed leaves by $18.3 \%$ and $48.8 \%$, respectively, compared with drought treatment alone (Fig. 3A, B). However, after 5 days of drought exposure, the ATPS activity in the sulfate + drought group was $19.2 \%$ lower than that in the drought group (Fig. $3 \mathrm{C}$ ). At the initiation of drought treatment $(0 \mathrm{~d})$, sulfate application did not change the activity of these three enzymes (Fig. 3A-C). The Cys content was markedly elevated in the leaves of foxtail millet seedlings exposed to 5 days of drought treatment relative to the control (Fig. 3D). Under drought conditions, sulfate pretreatment significantly increased the Cys content by $21.3 \%$ compared with non-sulfate-treated seedlings (Fig. 3D). The transcript expression of the SISIR, SiOASTL, and SiATPSgenes was also examined in the leaves of drought-stressed seedlings. Five days of drought treatment resulted in a remarkable increase in the expression level of SiSiR compared with the control, while for SiOASTL gene expression, there was no significant difference between the two groups (Fig. 4A, B). Notably, sulfate pretreatment significantly upregulated the expression of SiSiR and SIOASTL by $35.5 \%$ and $70.8 \%$, respectively, compared with drought treatment alone (Fig. 4A, B). However, the transcript expression of the SiATPS gene was dramatically inhibited by sulfate application under drought conditions (Fig. 4C).

\section{Sulfate application promoted ABA biosynthesis in drought- stressed foxtail millet leaves}

To examine whether sulfate application affects $A B A$ biosynthesis, $A B A$ content as well as the transcript expression of genes related to ABA synthesis (SINCED3, SIABA3, and SiAAO3) was measured in the leaves of foxtail millet seedlings. Five days of drought stress significantly increased $A B A$ content in leaves compared with the control (Fig. 5A). The application of $4 \mathrm{mM}$ sulfate further increased ABA level in drought-stressed leaves by $51.5 \%$ in comparison with drought treatment alone (Fig. $5 \mathrm{~A}$ ). After 5 days of drought exposure, the transcript expression levels of SINCED3, SIABA3, and SIAAO3 were upregulated to varying degrees in comparison with the control (Fig. 5B-D). Under drought conditions, pretreatment with sulfate significantly increased the expression levels of $\operatorname{SiABA3}$ and $\operatorname{SiAAO}$ by $49.2 \%$ and $59.3 \%$, respectively, compared with drought alone (Fig. 5C, D). However, sulfate application did not change the transcript expression of SiNCED3 under either normal or drought conditions (Fig. 5B).

Sulfate induced stomatal closure and reduced leaf water loss in foxtail millet seedlings under drought stress

The impact of exogenously applied sulfate on stomatal movement was determined in the leaves of foxtail millet seedlings. Treatment with sulfate $(4 \mathrm{mM})$ efficiently induced stomatal closure under wellwatered and drought stress conditions (Fig. 6A). Upon drought exposure, sulfate pretreatment significantly reduced stomatal apertures by $26.3 \%$ compared with the control (Fig. 6B). This effect was strengthened when $5 \mu \mathrm{M}$ ABA was applied in combination with sulfate, with mean stomatal aperture $46.1 \%$ lower in the sulfate + ABA group than in the sulfate group (Fig. 6B). In addition, ABA applied alone also resulted in remarkable decline in stomatal aperture compared with the control, under both normal and drought conditions (Fig. 6B). 
To test whether stomatal closure was linked with lower water loss during drought exposure, we measured leaf water loss from detached leaves of foxtail millet seedlings grown under drought conditions. As shown in Fig. 6C, $4 \mathrm{mM}$ sulfate application decreased the water loss rate compared with the control. After $2 \mathrm{~h}$ of air exposure, the water lost by the leaves of sulfate-pretreated seedlings was $14.5 \%$, while the water lost by the control leaves was $21.3 \%$ (Fig. 6C). A similar trend was observed in the detached leaves of seedlings pretreated with $5 \mu \mathrm{M}$ ABA (Fig. $6 \mathrm{C}$ ). It should be noted that detached leaves from seedlings subjected to a combined treatment of sulfate and ABA displayed the lowest rate of water loss $(13.6 \%$; Fig. 6C).

\section{Discussion}

As the main source of sulfur nutrition, sulfate plays significant roles in plant growth, metabolic processes, and stress signalling and responses (Davidian and Kopriva 2010; Kopriva et al. 2019). The present study focuses on the impact of exogenous sulfate application on drought stress mitigation in foxtail millet seedlings. Drought usually reduces tissue water content and thereby leads to metabolic impairment in plants (Xu et al. 2010). Our results revealed that $4 \mathrm{mM}$ sulfate rescued the decline in leaf relative water content induced by drought stress (Fig. 1), suggesting that sulfate is able to enhance the drought tolerance of foxtail millet. This finding is consistent with a previous report showing that sulfate application can improve the drought tolerance of maize plants (Usmani et al. 2020). The possible mechanisms of sulfate-enhanced drought tolerance were subsequently investigated in foxtail millet seedlings.

Drought exposure reduced sulfate contents in foxtail millet leaves (Fig. 2). Similarly, Ahmad et al. (2016) previously reported that drought-stressed maize plants accumulated less sulfate in the leaves than did well-watered controls. One possible explanation for this pattern may be the decreased root-to-shoot sulfate transport during drought. Drought-triggered stomatal closure is thought to limit the xylem transpiration stream, thus reducing sulfate translocation to the aerial parts (Gallardo et al. 2014). Furthermore, the reallocation of sulfate from the shoot to root may partially contribute to the decreased sulfate contents observed in drought-stressed leaves, a potential mechanism which warrants further investigation. Exogenous sulfate application increased the sulfate levels within leaf tissues upon drought stress (Fig. 2). In plants, sulfur assimilation is modulated by sulfur availability. A high sulfate supply may enhance sulfur assimilation, which can stimulate the biosynthesis of Cys (Hirai et al. 2003; Chan et al. 2013). In the present study, we found that exogenous sulfate application increased the activity of two key enzymes involved in sulfur assimilation, SiR and OASTL, thereby improving Cys biosynthesis in foxtail millet leaves upon drought exposure (Fig. 3). However, in contrast to previously reported results in heavy metal-stressed Populus deltoides and Brassica chinensis (Liang et al. 2016; Ma et al. 2018), we noticed that both ATPS enzyme activity and gene expression level were downregulated in drought-stressed leaves with sulfate application (Figs. 3 and 4). We assume that the accumulation of Cys may exert feedback inhibition on ATPS transcript abundance and protein levels, as studies in Arabidopsis thaliana have revealed that ATPS mRNA, protein and enzyme activity could be decreased by exogenous Cys application (Vauclare et al. 2002). 
Cys serves as the sulfur donor for molybdenum cofactor (Moco) sulfuration by Moco sulfurase (ABA3), which is required for the activation of abscisic aldehyde oxidase 3 (AAO3), a key enzyme in ABA biosynthesis (Bittner et al. 2001). Here, external sulfate supply upregulated the expression of ABA3 and AAO3 genes and thus increased the ABA levels within drought-stressed foxtail millet leaves (Fig. 5). These changes may be attributed to the increased Cys production elicited by sulfate application under drought conditions. In Arabidopsis, sulfate-depleted mutants have been found to show significantly lower total AAO (including AAO3) activity than wild-type plants. Remarkably, exogenous application of Cys was able to restore $A A O$ activity and facilitate $A B A$ synthesis in the mutants (Cao et al. 2014). Furthermore, xanthoxin production catalysed by NCED is also a limiting step for ABA biosynthesis (Milborrow 2001). Batool et al. (2018) reported that petiole feeding of sulfate or Cys stimulated ABA synthesis by inducing NCED3 transcription in Arabidopsis leaves. However, in the present study, sulfate supply did not change SiNCED3 transcript expression in foxtail millet leaves (Fig. 5). These findings suggest that Cys-induced $A B A$ biosynthesis depends on the activation of $A B A 3$ and $A A O 3$ in foxtail millet leaves under drought stress.

Sulfate has been reported to stimulate stomatal closure in several plant species, including poplar, wheat and Arabidopsis (Malcheska et al. 2017; Chen et al. 2019; Rajab et al. 2019). For the induction of stomatal closure, sulfate needs to be incorporated into Cys to trigger ABA biosynthesis (Batool et al. 2018). Consequently, ABA synthesis-depleted mutants fail to close stomata following sulfate application (Batool et al. 2018). In this work, we also demonstrated that sulfate application can induce stomatal closure in foxtail millet leaves, and this effect can be enhanced by exogenous ABA application (Fig. 6). A large number of previous studies have highlighted the importance of stomata in limiting transpirational water loss under dehydration conditions (Basu et al. 2016; Gupta et al. 2020). The results of the current study demonstrate that sulfate application lowered water loss and increased water content in response to dehydration stress (Figs. 1 and 6). It is assumed that the stomatal closure induced by sulfate supply may enhance ability to retain water status in foxtail millet leaves under drought stress. Overall, these results led us to propose that sulfate can improve drought tolerance in foxtail millet plants, at least partially, by promoting $A B A$ biosynthesis and inducing stomatal closure. However, the stimulatory effect of sulfate on ABA synthesis might not be solely responsible for sulfate-induced stomatal closure upon drought. Previous studies with poplar have revealed that sulfate can directly activate the R-type/quick-activating anion channel (QUAC1) on guard cell plasma membranes, leading to $\mathrm{K}^{+}$release and thus stomatal closure (Malcheska et al. 2017). Certainly, the detailed molecular mechanisms underlying sulfateenhanced drought tolerance in foxtail millet plants need to be investigated in the future using reverse genetic approaches.

\section{Conclusions}

In summary, we proposed a schematic model explaining the mechanisms underlying sulfate-promoted drought tolerance in foxtail millet seedlings (Fig. 7). Under water deficit stress, exogenous sulfate application enhanced sulfur assimilation by promoting activity of the enzymes SiR and OASTL, thus 
triggering increased Cys production. The elevated Cys upregulated the expression of the $S A B B A 3$ and SIAAO3 genes and stimulated ABA biosynthesis, which induced stomatal closure and reduced water loss in drought-stressed foxtail millet leaves. Taken together, our data support the hypothesis that under drought stress conditions, exogenously applied sulfate may be incorporated into Cys to trigger ABA biosynthesis and promote stomatal closure, contributing to the water loss limitation response in foxtail millet leaves.

\section{Declarations}

\section{Conflicts of interest}

The authors declare that they have no conflicts of interest.

\section{Acknowledgements}

This research was supported by the Natural Science Foundation Project of Shanxi Province of China (201901D211181, 201801D221126), the National Natural Science Foundation of China (31500504, 31972132), Scientific and Technological Innovation Programs of Higher Education Institutions in Shanxi (2017102).

\section{References}

Ahmad N, Malagoli M, Wirtz M, Hell R (2016) Drought stress in maize causes differential acclimation responses of glutathione and sulfur metabolism in leaves and roots. BMC Plant Biol 16:247.

Basu S, Ramegowda V, Kumar A, Pereira A (2016) Plant adaptation to drought stress. F1000Res 5:1554.

Batool S, Uslu VV, Rajab H, Ahmad N, Waadt R, Geiger D, Malagoli M, Xiang CB, Hedrich R, Rennenberg $H$, Herschbach C, Hell R, Wirtz M (2018) Sulfate is incorporated into cysteine to trigger ABA production and stomatal closure. Plant Cell 30:2973-2987.

Bittner F, Oreb M, Mendel RR (2001) ABA3 is a molybdenum cofactor sulfurase required for activation of aldehyde oxidase and xanthine dehydrogenase in Arabidopsis thaliana. J Biol Chem 276:40381-40384.

Bradford MM (1976) A rapid and sensitive method for the quantitation of microgram quantities of protein utilizing the principle of protein-dye binding. Anal Biochem 72:248-254.

Cao MJ, Wang Z, Zhao Q, Mao JL, Speiser A, Wirtz M, Hell R, Zhu JK, Xiang CB (2014) Sulfate availability affects $\mathrm{ABA}$ levels and germination response to ABA and salt stress in Arabidopsis thaliana. Plant $\mathrm{J}$ 77:604-615. 
Chan KX, Wirtz M, Phua SY, Estavillo GM, Pogson BJ (2013) Balancing metabolites in drought: the sulfur assimilation conundrum. Trends Plant Sci 18:18-29.

Chen Z, Zhao PX, Miao ZQ, Qi GF, Wang Z, Yuan Y, Ahmad N, Cao MJ, Hell R, Wirtz M, Xiang CB (2019) SULTR3s function in chloroplast sulfate uptake and affect ABA biosynthesis and the stress response. Plant Physiol 180:593-604.

Davidian JC, Kopriva S (2010) Regulation of sulfate uptake and assimilation-the same or not the same? Mol Plant 3:314-325.

Du C, Chai L, Wang Z, Fan H (2019) Response of proteome and morphological structure to short-term drought and subsequent recovery in Cucumis sativus leaves. Physiol Plant 167:676-689.

Ernst L, Goodger JQD, Alvarez S, Marsh EL, Berla B, Lockhart E, Jung J, Li P, Bohnert HJ, Schachtman DP (2010) Sulphate as a xylem-borne chemical signal precedes the expression of ABA biosynthetic genes in maize roots. J Exp Bot 61:3395-3405.

Fang Q, Jiang T, Xu L, Liu H, Mao H, Wang X, Jiao B, Duan Y, Wang Q, Dong Q, Yang L, Tian G, Zhang C, Zhou Y, Liu X, Wang H, Fan D, Wang B, Luo K (2017) A salt-stress-regulator from the Poplar R2R3 MYB family integrates the regulation of lateral root emergence and ABA signaling to mediate salt stress tolerance in Arabidopsis. Plant Physiol Biochem 114:100-110.

Gaitonde MK (1967) A spectrophotometric method for the direct determination of cysteine in the presence of other naturally occurring amino acids. Biochem J 104:627-633.

Gallardo K, Courty PE, Le Signor C, Wipf D, Vernoud V (2014) Sulfate transporters in the plant's response to drought and salinity: regulation and possible functions. Front Plant Sci 5:580.

Gupta A, Rico-Medina A, Caño-Delgado Al (2020) The physiology of plant responses to drought. Sci 368:266-269.

Han Y, Yang H, Wu M, Yi H (2019) Enhanced drought tolerance of foxtail millet seedlings by sulfur dioxide fumigation. Ecotoxicol Environ saf 178:9-16.

Hao X, Jin Z, Wang Z, Qin W, Pei Y (2020) Hydrogen sulfide mediates DNA methylation to enhance osmotic stress tolerance in Setaria italica L. Plant Soil 453:355-370.

Hirai MY, Fujiwara T, Awazuhara M, Kimura T, Noji M, Saito K (2003) Global expression profiling of sulfurstarved Arabidopsis by DNA macroarray reveals the role of $O$-acetyl-L-serine as a general regulator of gene expression in response to sulfur nutrition. Plant $\mathrm{J}$ 33:651-663.

Kopriva S, Malagoli M, Takahashi H (2019) Sulfur nutrition: impacts on plant development, metabolism, and stress responses. J Exp Bot 70:4069-4073. 
Koralewska A, Buchner P, Stuiver CEE, Posthumus FS, Kopriva S, Hawkesford MJ, De Kok LJ (2009) Expression and activity of sulfate transporters and APS reductase in curly kale in response to sulfate deprivation and re-supply. J Plant Physiol 166:168-179.

Liang T, Ding H, Wang G, Kang J, Pang H, Lv J (2016) Sulfur decreases cadmium translocation and enhances cadmium tolerance by promoting sulfur assimilation and glutathione metabolism in Brassica chinensis L. Ecotoxicol Environ Saf 124:129-137.

Lim CW, Baek W, Jung J, Kim JH, Lee SC (2015) Function of ABA in stomatal defense against biotic and drought stresses. Int J Mol Sci 16:15251-15270.

Livak KJ, Schmittgen TD (2001) Analysis of relative gene expression data using real-time quantitative PCR and the $2^{-\Delta \Delta C t}$ method. Methods 25:402-408.

Ma C, Chen Y, Ding S, Li Z, Shi WG, Zhang Y, Luo ZB (2018) Sulfur nutrition stimulates lead accumulation and alleviates its toxicity in Populus deltoides. Tree Physiol 38:1724-1741.

Malcheska F, Ahmad A, Batool S, Müller HM, Ludwig-Müller J, Kreuzwieser J, Randewig D, Hänsch R, Mendel RR, Hell R, Wirtz M, Geiger D, Ache P, Hedrich R, Herschbach C, Rennenberg H (2017) Droughtenhanced xylem sap sulfate closes stomata by affecting ALMT12 and guard cell ABA synthesis. Plant Physiol 174:798-814.

Milborrow BV (2001) The pathway of biosynthesis of abscisic acid in vascular plants: a review of the present state of knowledge of ABA biosynthesis. J Exp Bot 52:1145-1164.

Pant SR, Irigoyen S, Doust AN, Scholthof KBG, Mandadi KK (2016) Setaria: a food crop and translational research model for $\mathrm{C}_{4}$ grasses. Front Plant Sci 7:1885.

Qi J, Song CP, Wang B, Zhou J, Kangasjärvi J, Zhu JK, Gong Z (2018) Reactive oxygen species signaling and stomatal movement in plant responses to drought stress and pathogen attack. J Integr Plant Biol 60:805-826.

Rajab H, Khan MS, Malagoli M, Hell R, Wirtz M (2019) Sulfate-induced stomata closure requires the canonical ABA signal transduction machinery. Plants 8:21.

Schachtman DP, Goodger JQD (2008) Chemical root to shoot signaling under drought. Trends Plant Sci 13:281-287.

Takahashi H (2019) Sulfate transport systems in plants: functional diversity and molecular mechanisms underlying regulatory coordination. J Exp Bot 70:4075-4087.

Tang S, Li L, Wang Y, Chen Q, Zhang W, Jia G, Zhi H, Zhao B, Diao X (2017) Genotype-specific physiological and transcriptomic responses to drought stress in Setaria italica (an emerging model for Panicoideae grasses). Sci Rep 7:10009. 
Usmani MM, Nawaz F, Majeed S, Shehzad MA, Ahmad KS, Akhtar G, Aqib M, Shabbir RN (2020) Sulfatemediated drought tolerance in maize involves regulation at physiological and biochemical levels. Sci Rep 10:1147.

Vauclare P, Kopriva S, Fell D, Suter M, Sticher L, von Ballmoos P, Krähenbühl U, den Camp RO, Brunold C (2002) Flux control of sulphate assimilation in Arabidopsis thaliana: adenosine 5'-phosphosulphate reductase is more susceptible than ATP sulphurylase to negative control by thiols. Plant $J$ 31:729-740.

Xu Z, Zhou G, Shimizu H (2010) Plant responses to drought and rewatering. Plant Signal Behav 5:649654.

Yang Z, Zhang H, Li X, Shen H, Gao J, Hou S, Zhang B, Mayes S, Bennett M, Ma J, Wu C, Sui Y, Han Y, Wang X (2020) A mini foxtail millet with an Arabidopsis-like life cycle as a $\mathrm{C}_{4}$ model system. Nat Plants 6:1167-1178.

Zhang G, Liu X, Quan Z, Cheng S, Xu X, Pan S, Xie M, Zeng P, Yue Z, Wang W, Tao Y, Bian C, Han C, Xia Q, Peng X, Cao R, Yang X, Zhan D, Hu J, Zhang Y, Li H, Li H, Li N, Wang J, Wang C, Wang R, Guo T, Cai Y, Liu C, Xiang H, Shi Q, Huang P, Chen Q, Li Y, Wang J, Zhao Z, Wang J (2012a) Genome sequence of foxtail millet (Setaria italica) provides insights into grass evolution and biofuel potential. Nat Biotechnol 30:549554.

Zhang K, Xia X, Zhang Y, Gan SS (2012b) An ABA-regulated and golgi-localized protein phosphatase controls water loss during leaf senescence in Arabidopsis. Plant J 69:667-678.

\section{Figures}



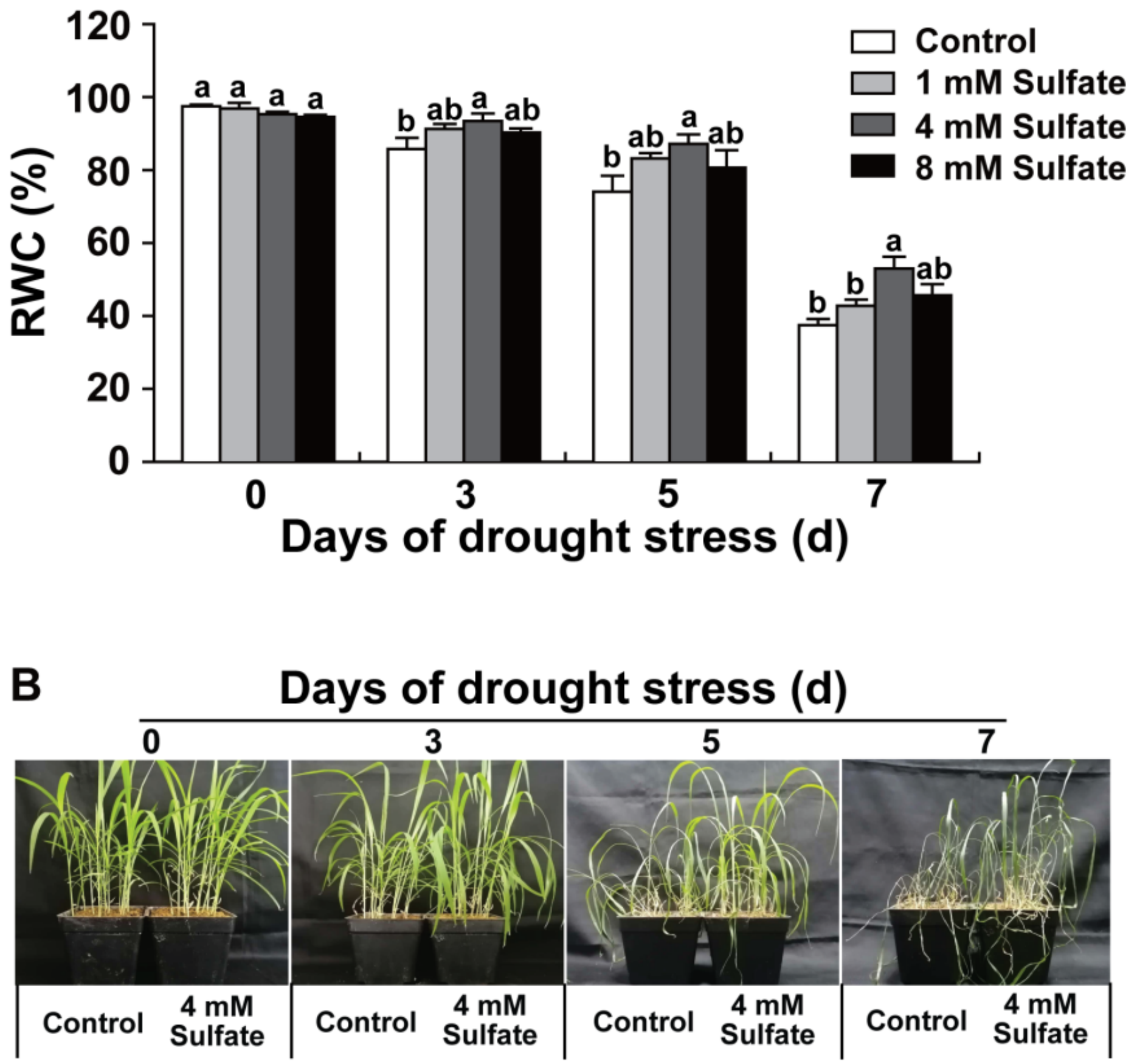

Figure 1

Effects of exogenous sulfate application on the leaf RWC and phenotypes of foxtail millet seedlings under drought stress. Three-week-old seedlings were pretreated with water (control) or the indicated concentrations of MgSO4 solution (sulfate) and subsequently subjected to drought by terminating water supply. After $0,3,5$, and 7 days of drought stress, leaf RWC (A) was measured, and the phenotypes of seedlings (B) were recorded. At each time point, different letters indicate statistically significant differences at $\mathrm{P}<0.05$. 


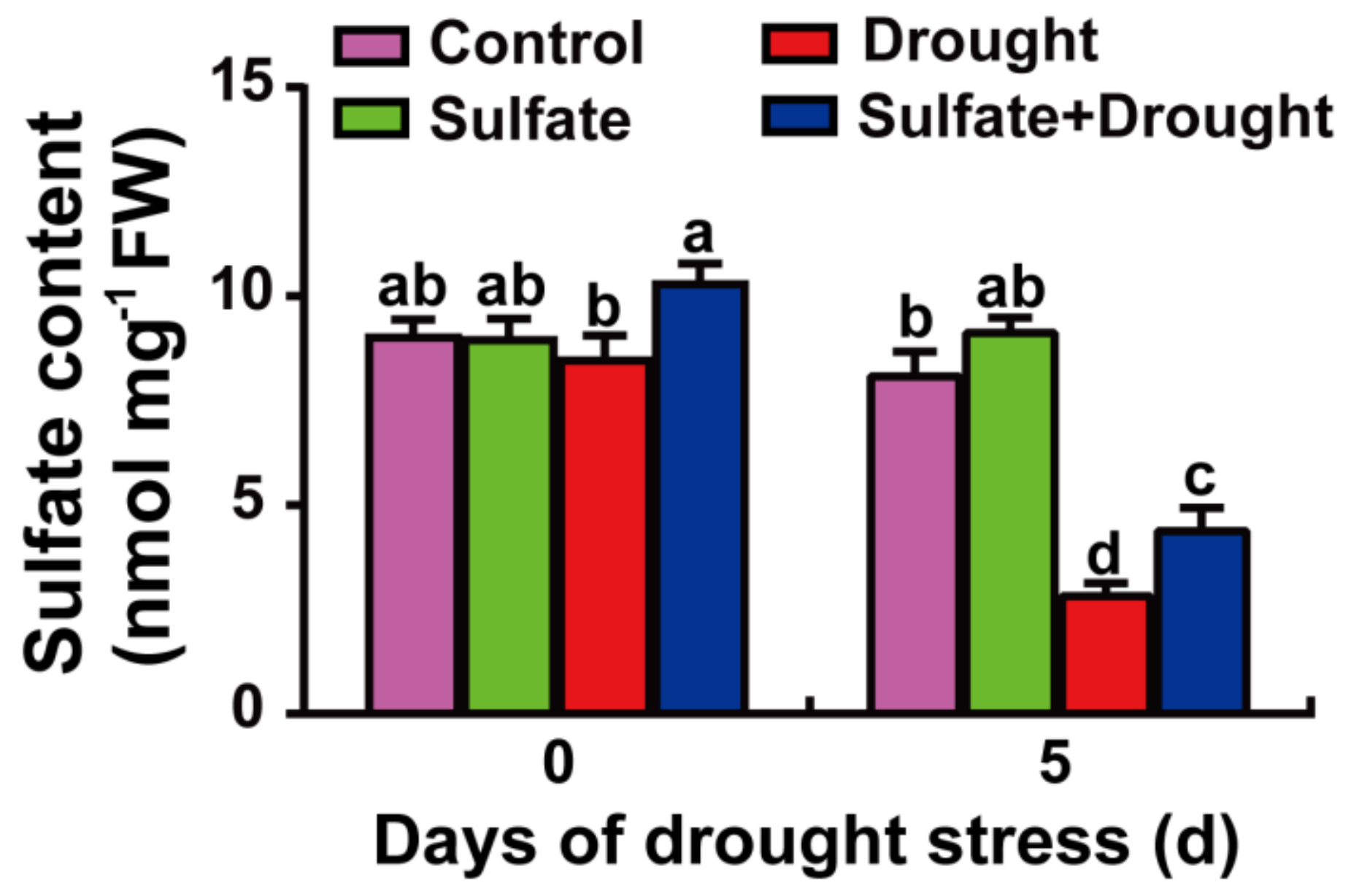

Figure 2

Endogenous sulfate content in the leaves of foxtail millet seedlings. Three-week-old seedlings were pretreated with water or $4 \mathrm{mM} \mathrm{MgSO} 4$ solution and then regularly watered or subjected to drought treatment by withholding water. Endogenous sulfate content in leaves was measured after 0 and 5 days of drought stress. Bars with different letters indicate significant differences at $P<0.05$. 

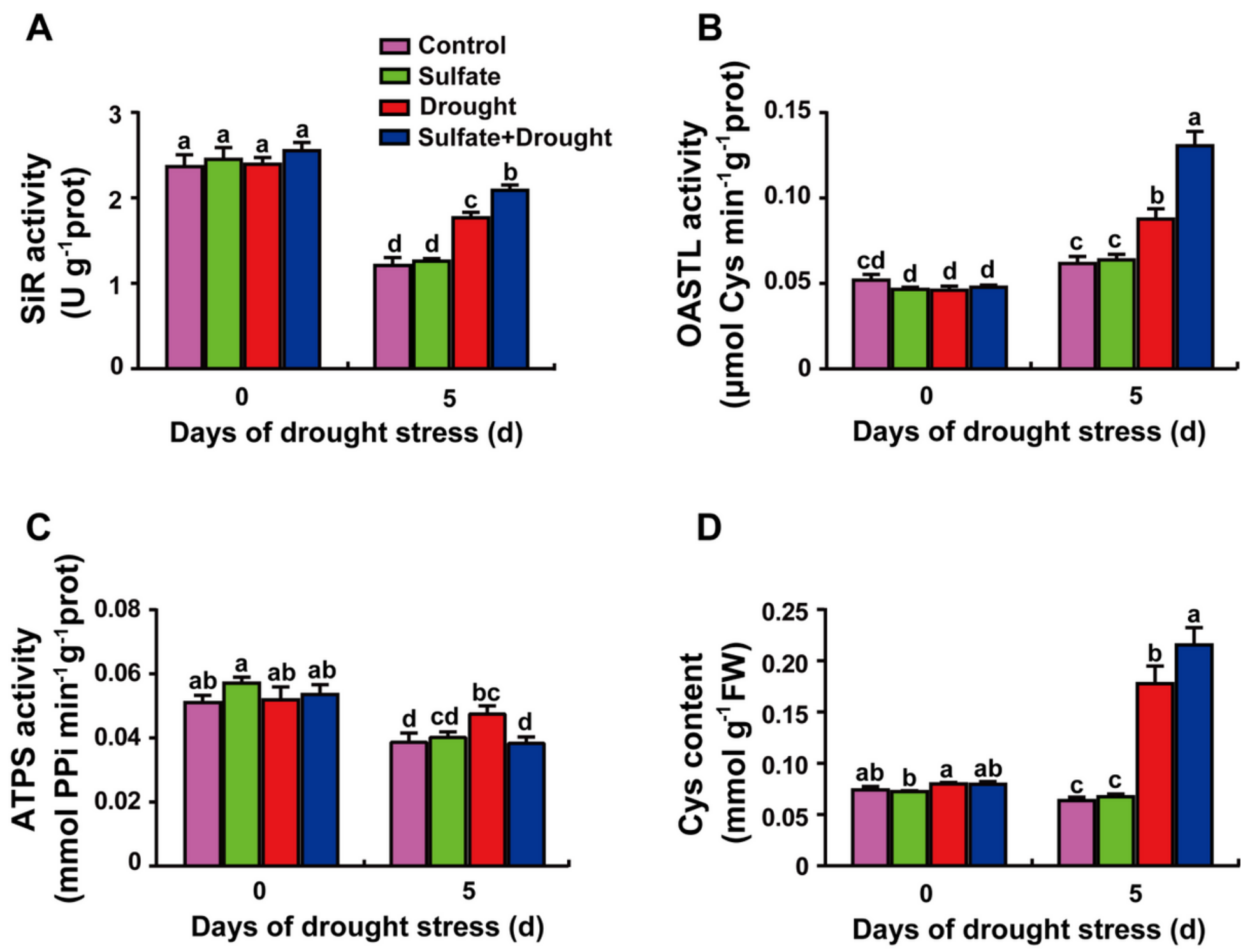

D

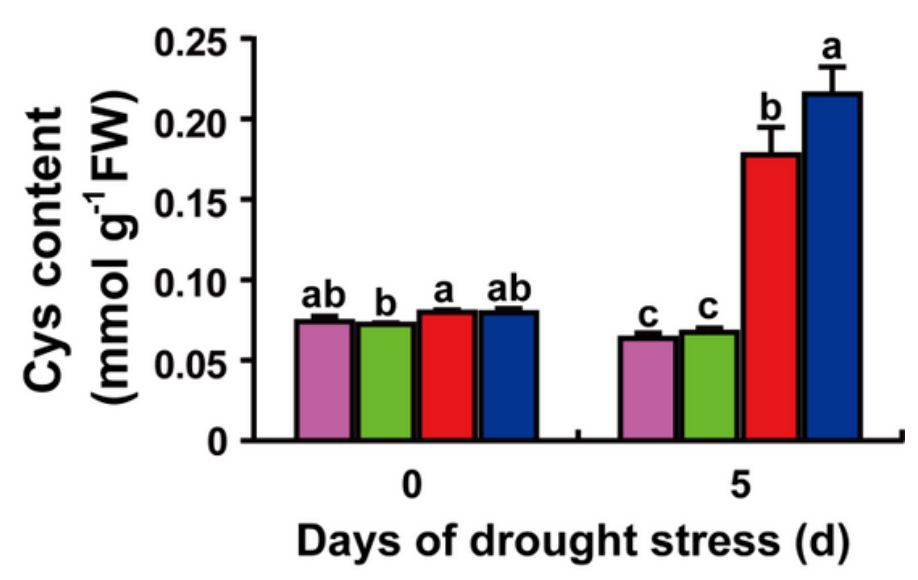

Figure 3

Effects of sulfate application on Cys content and SiR, OASTL, and ATPS activity in the leaves of foxtail millet seedlings under drought stress. Three-week-old seedlings were pretreated with water or $4 \mathrm{mM}$ MgSO4 solution and then regularly watered or subjected to drought treatment by withholding water. After 0 and 5 days of drought stress, the activity of SiR, OASTL, and ATPS and the content of Cys were measured $(A-D)$. Bars with different letters indicate significant differences at $P<0.05$. 


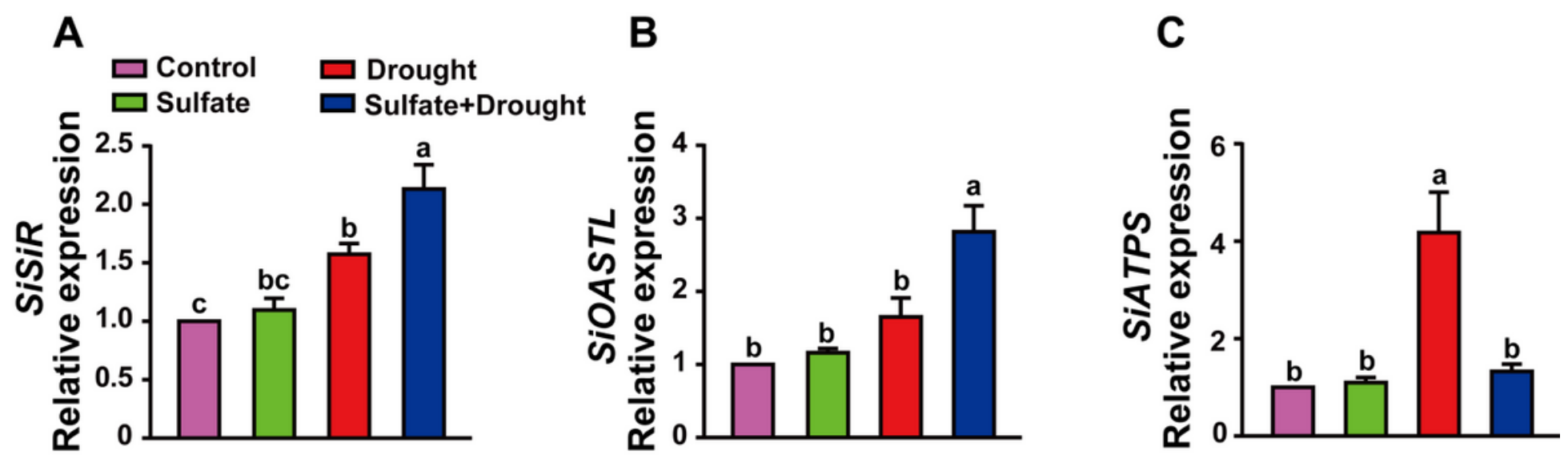

Figure 4

Effects of sulfate application on the transcriptional expression of SiSiR, SiOASTL, and SiATPS genes in the leaves of foxtail millet seedlings under drought stress. Three-week-old seedlings were pretreated with water or $4 \mathrm{mM} \mathrm{MgSO} 4$ solution and then regularly watered or subjected to drought treatment by withholding water. After 5 days of drought stress, the relative transcript expression levels of SiSiR, SiOASTL, and SiATPS were assayed by quantitative real-time PCR. Bars with different letters indicate significant differences at $\mathrm{P}<0.05$. 
A

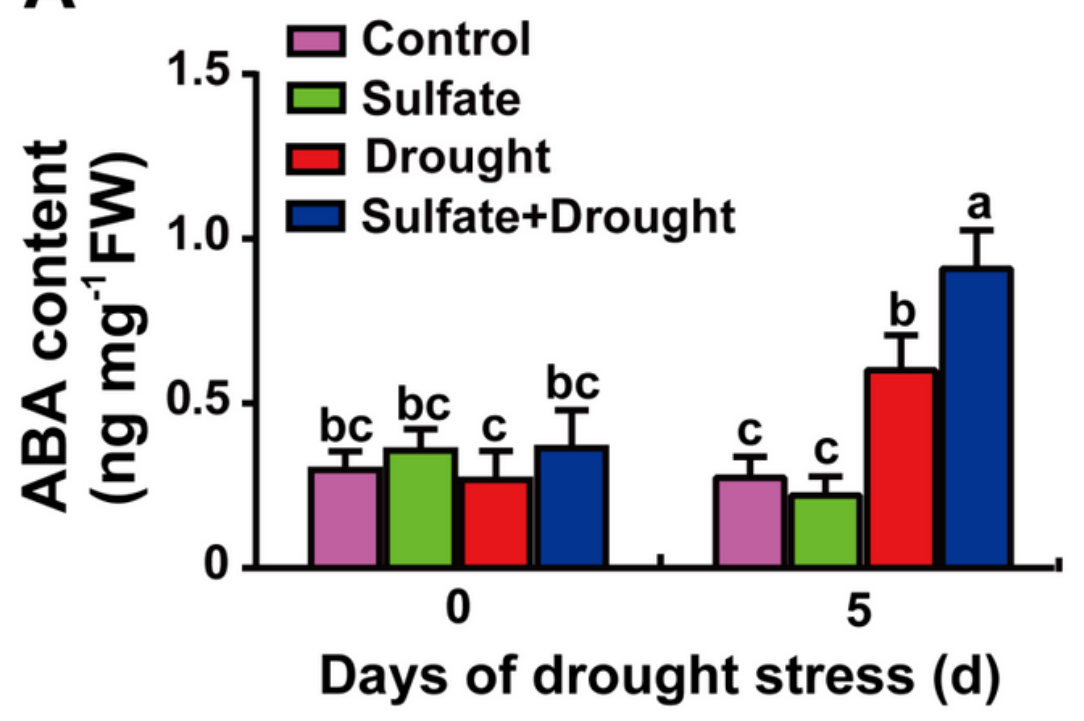

B

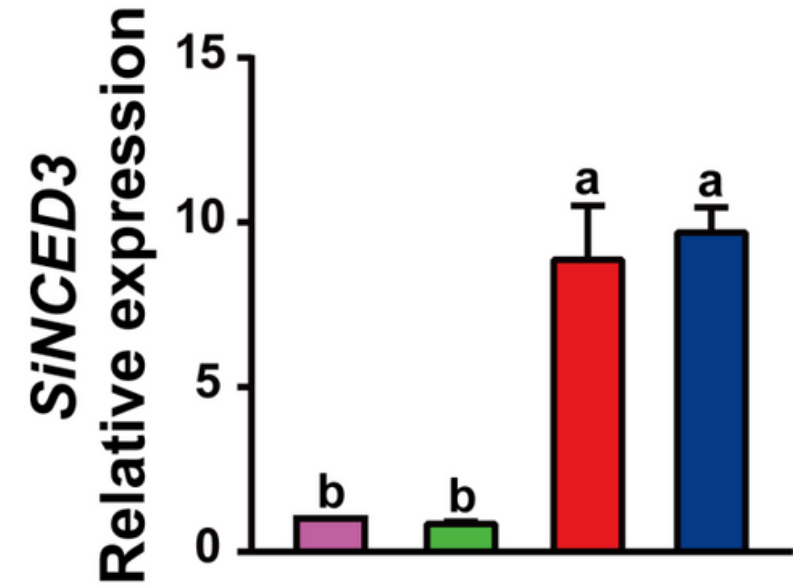

Days of drought stress (d)
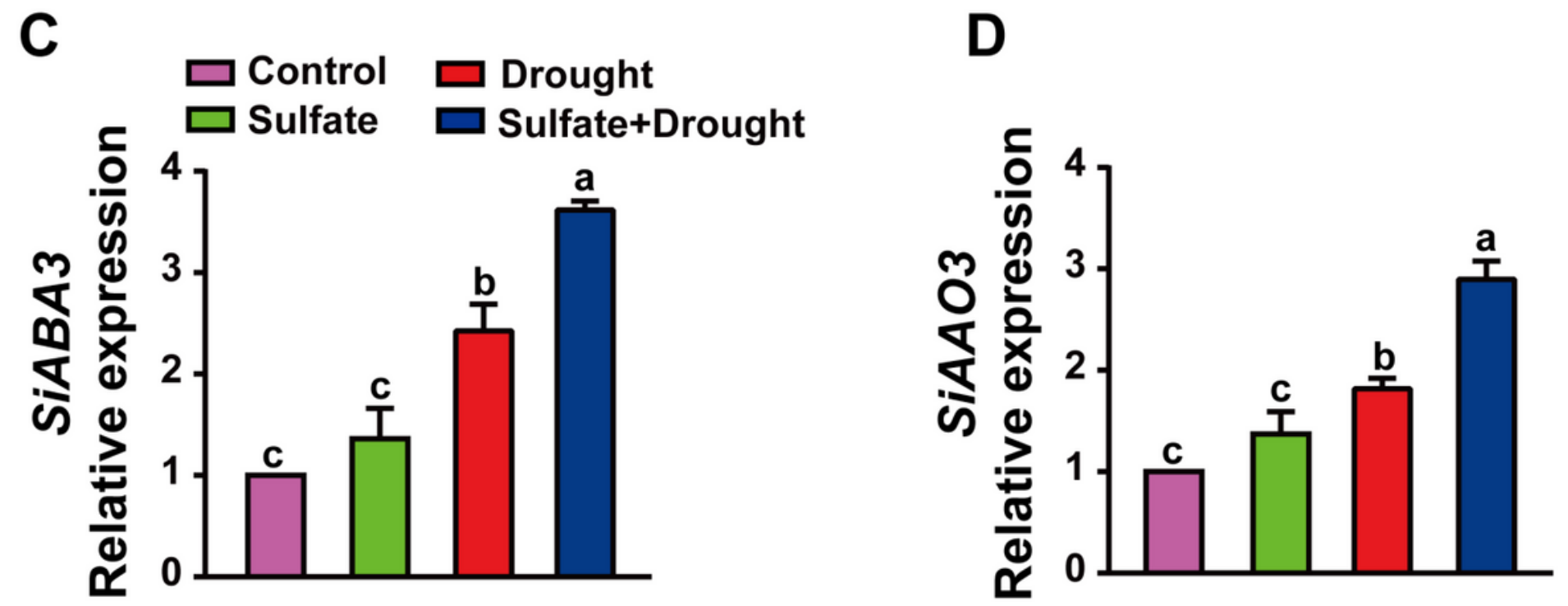

Figure 5

Effects of sulfate application on ABA content and transcript expression of genes involved in ABA biosynthesis in the leaves of drought-stressed seedlings. Three-week-old seedlings were pretreated with water or $4 \mathrm{mM} \mathrm{MgSO} 4$ solution and then regularly watered or subjected to drought treatment by withholding water. After 0 and 5 days of drought stress, ABA content was measured $(A)$. The relative expression levels of SiNCED3, SiABA3, and SiAAO3 were assayed after 5 days of drought exposure (B-D). Bars with different letters denote significant differences at $\mathrm{P}<0.05$. 
A

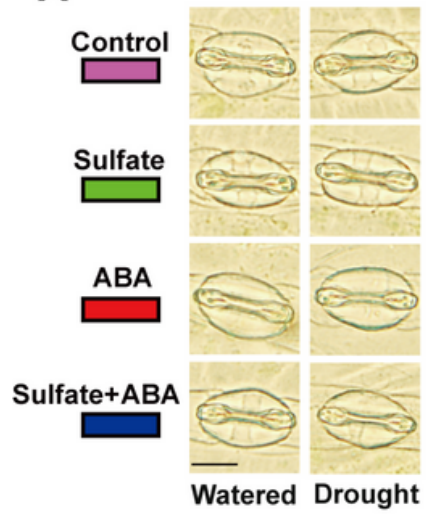

B

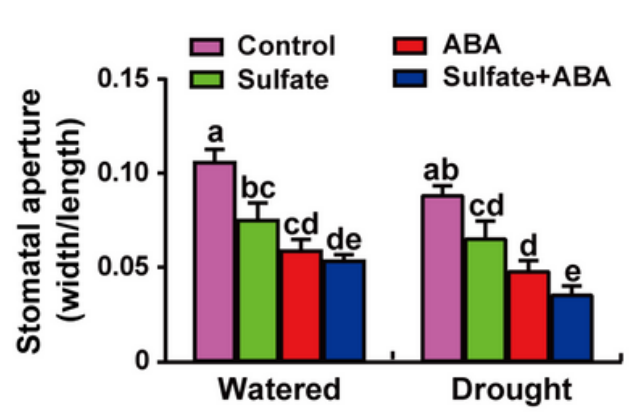

C

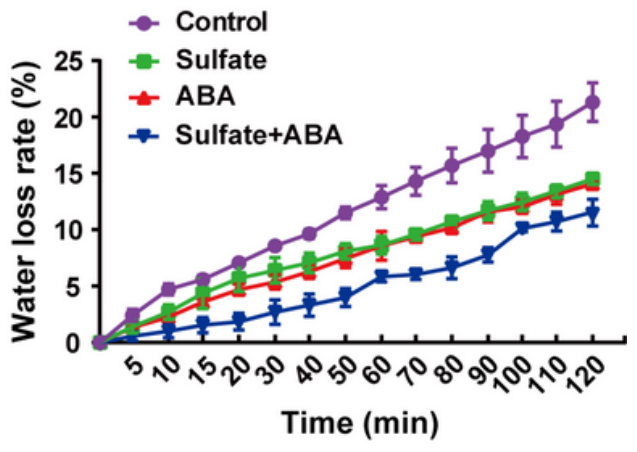

Figure 6

Effects of sulfate application on stomatal apertures and leaf water loss in foxtail millet seedlings under drought conditions. Three-week-old seedlings were pretreated with water (control), 4 mM MgSO4 (sulfate), $5 \mu \mathrm{M} \mathrm{ABA}$, or $4 \mathrm{mM}$ MgSO 4 plus $5 \mu \mathrm{M}$ ABA and then regularly watered (watered) or subjected to drought treatment (drought) by withholding water for 5 days. (A) Representative images of the stomata.

(B) Stomatal aperture. (C) Water loss of detached leaves from drought-stressed seedlings. Bars with different letters denote significant differences at $P<0.05$. Scale bar $=20 \mu \mathrm{m}$.

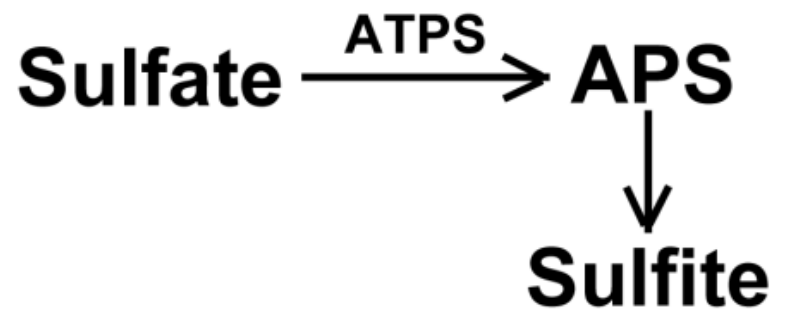

\section{SiR \\ OASTL}

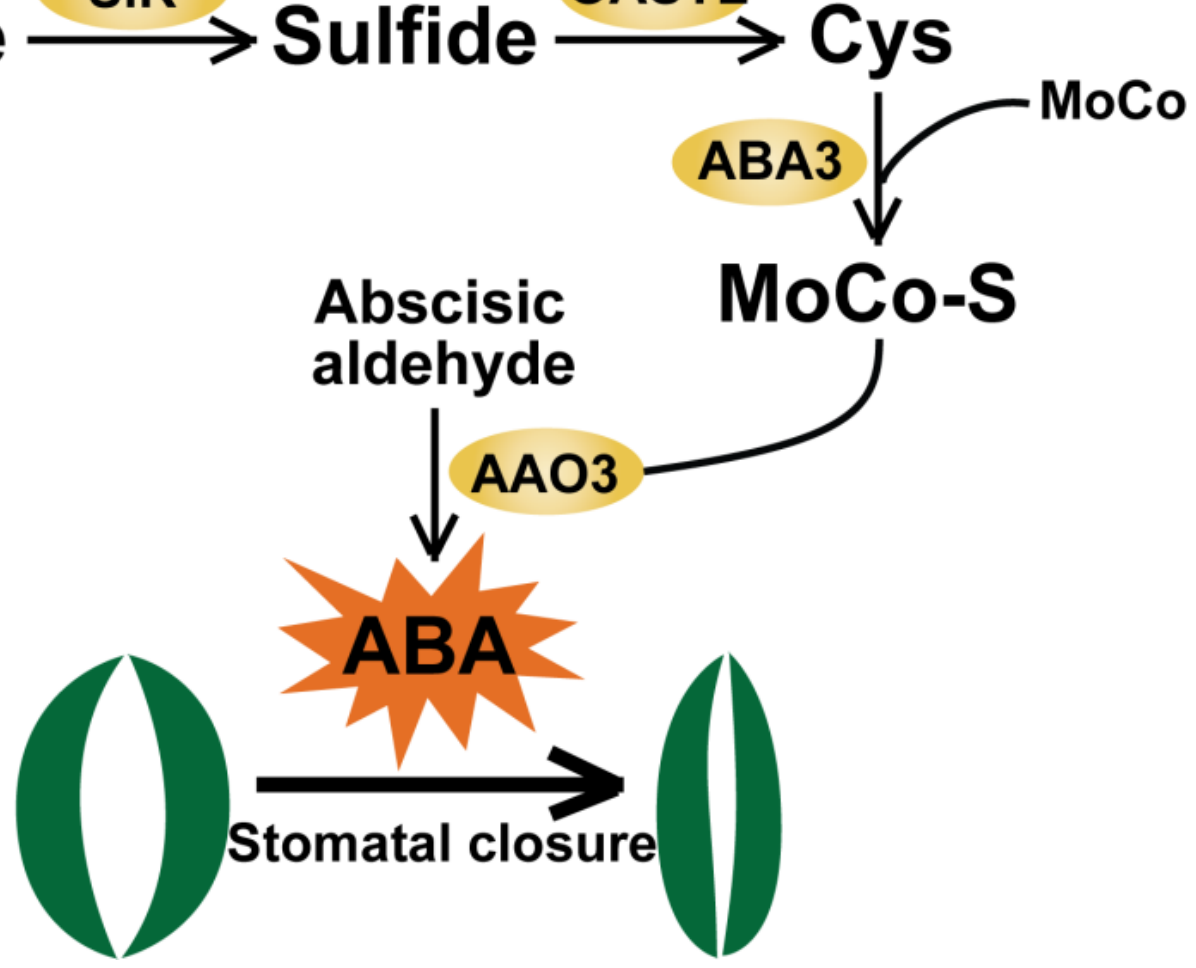

Figure 7 
Schematic model summarizing the effect of sulfate on ABA biosynthesis and stomatal closure in foxtail millet leaves under drought exposure. Yellow circles indicate enzymes activated by sulfate application.

\section{Supplementary Files}

This is a list of supplementary files associated with this preprint. Click to download.

- SupplementaryDataPS2021.docx 